Internat. J. Math. \& Math. Sci.

Vol. 22, No. 2 (1999) 251-258

S 0161-1712<99>22251-X

(c) Electronic Publishing House

\title{
TWO COUNTABLE, BICONNECTED, NOT WIDELY CONNECTED HAUSDORFF SPACES
}

\author{
V. TZANNES
}

(Received 5 November 1996 and in revised form 6 July 1998)

\begin{abstract}
We construct two countable, biconnected spaces, not widely connected, not having a dispersion point, and not being strongly connected. The first is Hausdorff and the second is Urysohn and almost regular.
\end{abstract}

Keywords and phrases. Countable connected, biconnected, almost regular.

1991 Mathematics Subject Classification. 54D05, 54D10, 54G15.

1. Introduction. The first example of a biconnected space with a dispersion point was constructed by B. Knaster and K. Kuratowski in [23], and the first example of a biconnected space without a dispersion point by E. W. Miller in [26]. Two stronger examples of biconnected spaces without a dispersion point were constructed by $\mathrm{M}$. E. Rudin in [30, 31]. The example in [30] has the property that the complement of every connected subset containing more than one point is at most countable and the example in [31] has the property of being widely connected. All spaces in [26, 30, 31], are subsets of the plane. The first two are constructed under the Continuum Hypothesis and the third one under Martin's Axiom. In [7], G. Gruenhage constructed a countable connected Hausdorff space under Martin's Axiom and a perfectly normal connected space under the Continuum Hypothesis in which the complement of every connected subspace containing more than one point is finite. In [36], we constructed a countable widely connected Hausdorff space and a countable widely connected and biconnected Hausdorff space.

Now, we construct two countable spaces which are biconnected without being widely connected and without a dispersion point. The first is Hausdorff, and the second is Urysohn almost regular. In addition, as it is the case with widely connected spaces and spaces with a dispersion point, both have the property of not being strongly connected [13]. The construction is based on a modification of [16] or [20]. It can be also based on [37]. From the construction, it follows that there exist $2^{c}$ non-homeomorphic such spaces.

A space $X$ is called

(1) Urysohn if every pair of distinct points of $X$ have disjoint closed neighborhoods.

(2) Almost regular if $X$ contains a dense subset at every point of which the space is regular.

A connected space $X$ is called

(1) Biconnected (K. Kuratowski [24]) if it admits no decomposition into two connected disjoint proper subsets containing more than one point. 
(2) Widely connected (P. M. Swingle [34]) if every connected subset, containing more than one point, is dense.

A point $x$ of a connected space $X$ is called

(1) A cut point if $X \backslash\{x\}$ is disconnected.

(2) A dispersion point if $X \backslash\{x\}$ is totally disconnected.

A connected space $(X, \tau)$ is called

(1) Maximal connected if, for every strictly finer topology $\sigma$, the space $(X, \sigma)$ is not connected.

(2) Strongly connected if it has a finer maximal connected topology.

Biconnected spaces (countable or not, with or without a dispersion point) are considered in [26, 37, 1, 2, 3, 4, 6, 9, 10, 11, 18, 19, 21, 22, 25, 27, 28, 29, 33, 38, 39] and maximal connected spaces in $[13,1,5,8,12,14,15,32,35]$.

\section{Results}

THE SPACE $T$. For the construction of the countably, biconnected and not widely connected Hausdorff space $T$, we first construct an appropriate countable Hausdorff totally disconnected space $X$ containing a specific point $p$ and a closed discrete subspace $\mathbb{N}$ which cannot be separated by disjoint open sets. Then keeping fixed the subspace $\mathbb{N}$ and condensing the point $p$ (instead of condensing pairs of points as in $[16,20]$, or [37]), we construct the space $T$.

On the set

$$
X=\left\{a_{k i}: k, i=1,2, \ldots\right\} \cup \mathbb{N} \cup\{p\},
$$

where $\mathbb{N}$ is the space of natural numbers, we define the following topology: every point $a_{k i}$ is isolated. For the points of $\mathbb{N}$ a basis of open neighborhoods in $X$ is defined as follows: let $\mathscr{P}$ be a free ultrafilter on $\mathbb{N}$ and let $\mathscr{P}_{k}$ be the copy of $\mathscr{P}$ in $\left\{a_{k i}: i=1,2, \ldots\right\}$. If $U \in \mathscr{P}$, we denote the copy of $U$ in $\left\{a_{k i}: i=1,2, \ldots\right\}$ by $U_{k}$. Then, for every $k \in \mathbb{N}$, a basis of open neighborhoods is the collection of sets

$$
U(k)=\{k\} \cup\left\{a_{k i}: a_{k i} \in U_{k}\right\}, \quad U \in \mathscr{P} .
$$

For the point $p$, a basis of open neighborhoods is the collection of sets

$$
U(p)=\{p\} \cup\left\{a_{k i}: k \in U\right\}, \quad U \in \mathscr{P} .
$$

Obviously, the space $X$ is Hausdorff and totally disconnected but not regular since the point $p$ and the closed subset $\mathbb{N}$ cannot be separated by disjoint open sets.

We observe that every basic open neighborhood of $p$ is defined by some $U \in \mathscr{P}$, and every $U \in \mathscr{P}$ defines a basic open neighborhood $U(p)$. Obviously, $\overline{U(p)} \backslash U(p)=U$.

Let $X^{1}(n), n=1,2, \ldots$ be disjoint copies of $X$ and let $\mathbb{N}^{1}(n)$ and $p^{1}(n)$ be the copies of $\mathbb{N}$ and $p$, respectively, in $X^{1}(n)$. The copies of $U(k)$ and $U(p)$ in $X^{1}(n)$ are denoted by $U\left(k^{1}(n)\right)$ and $U\left(p^{1}(n)\right)$, respectively. Since the set $P^{1}=\left\{p^{1}(n): n=1,2, \ldots\right\}$ and the dense subset $D=X \backslash \mathbb{N} \cup\{p\}$ of isolated points of $X$ are countable, there exists one-to-one function $f_{1}$ of $P^{1}$ onto $D$. We attach the spaces $X^{1}(n), n=1,2, \ldots$ to the space $X$ identifying simultaneously each point $p^{1}(n)$ with the point $f_{1}\left(p^{1}(n)\right)$ of $D$ and each set $\mathbb{N}^{1}(n)$ with $\mathbb{N}$ (by putting $k^{1}(n)$ on $k$ ). 
On the set

$$
T^{1}=X \cup \bigcup_{n=1}^{\infty}\left(X^{1}(n) \backslash\left(\mathbb{N}^{1}(n) \cup\left\{p^{1}(n)\right\}\right)\right),
$$

we define the following topology: every point of $T^{1} \backslash X$ is isolated. For every $k \in \mathbb{N}$, a basis of open neighborhoods is the collection of sets

$$
\begin{aligned}
O_{U}^{1}(k)=U(k) & \cup \bigcup_{n=1}^{\infty}\left(U\left(k^{1}(n)\right) \backslash\left\{k^{1}(n)\right\}\right) \\
& \cup \bigcup_{f_{1}\left(p^{1}(j)\right) \in U(k)}\left(U\left(p^{1}(j)\right) \backslash\left\{p^{1}(j)\right\}\right), \quad U \in \mathscr{P} .
\end{aligned}
$$

For every isolated point $x$ of $X$, a basis of open neighborhoods is the collection of sets

$$
O_{U}^{1}(x)=\{x\} \cup\left(U\left(p^{1}(j)\right) \backslash\left\{p^{1}(j)\right\}\right), \quad U \in \mathscr{P},
$$

where $f_{1}\left(p^{1}(j)\right)=x$.

For the point $p$, a basis of open neighborhoods is the collection of sets

$$
O_{U}^{1}(p)=U(p) \cup \underset{f_{1}\left(p^{1}(j)\right) \in U(p)}{\bigcup}\left(U\left(p^{1}(j)\right) \backslash\left\{p^{1}(j)\right\}\right), \quad U \in \mathscr{P} .
$$

It can be easily proved that the space $T^{1}$ is Hausdorff, totally disconnected, and contains the space $X$ as a closed nowhere dense subset. We observe that every basic open neighborhood in $T^{1}$, of every $x \in X$ is defined by some $U \in \mathscr{P}$, and every $U \in \mathscr{P}$ defines in $T^{1}$, a basic open neighborhood $O_{U}^{1}(x)$, for every $x \in X$. Obviously, $\overline{O_{U}^{1}(x)} \backslash O_{U}^{1}(x)=U$. Furthermore, for every pair of points $x, y$ of $D$ and every basic open neighborhoods $O_{U}^{1}(x), O_{V}^{1}(y), U, V \in \mathscr{P}$, of $x, y$ respectively, in $T^{1}$, it holds that $\overline{O_{U}^{1}(x)} \cap \overline{O_{V}^{1}(y)} \neq \varnothing$, which implies that every continuous real-valued function of $T^{1}$ is constant on $D$ and, hence, on $X$ since $D$ is dense in $X$.

We construct by induction the spaces $T^{2}, T^{3}, \ldots, T^{m}$, where

$$
T^{m}=T^{m-1} \cup \bigcup_{n=1}^{\infty}\left(X^{m-1}(n) \backslash\left(\mathbb{N}^{m-1}(n) \cup\left\{p^{m-1}(n)\right\}\right)\right),
$$

and where $X^{m-1}(n), n=1,2, \ldots$ are disjoint copies of the initial space $X$, and $\mathbb{N}^{m-1}(p)$, $P^{m-1}(n)$ are the copies of $\mathbb{N}, p$ in $X^{m-1}(n)$, respectively. Each point $p^{m-1}(n)$ is identified with the point $f_{m-1}\left(p^{m-1}(n)\right)$, where $f_{m-1}$ is one-to-one function of the set $P^{m-1}=\left\{p^{m-1}(n): n=1,2, \ldots\right\}$ onto the dense subset of isolated points of $T^{m-1}$. Each set $N^{m-1}(n)$ is identified with the set $\mathbb{N}$ (by putting $k^{m-1}(n)$ on $k$ ).

It can be easily proved that the space $T^{m}$ is Hausdorff, totally disconnected, and contains the space $T^{m-1}$ as a closed nowhere dense subset. We observe that every basic open neighborhood in $T^{m}$, of every $x \in T^{m-1}$ is defined by some $U \in \mathscr{P}$, and every $U \in$ $\mathscr{P}$, defines in $T^{m}$, a basic open neighborhood $O_{U}^{m}(x)$, for every $x \in T^{m-1}$. Obviously, $\overline{O_{U}^{m}(x)} \backslash O_{U}^{m}(x)=U$. Furthermore, for every pair $x, y$ of isolated points of $T^{m-1}$ and every basic open neighborhood $O_{U}^{m}(x), O_{V}^{m}(y), U, V \in \mathscr{P}$ of $x, y$ respectively, in $T^{m}$, 
it holds that $\overline{O_{U}^{m}(x)} \cap \overline{O_{V}^{m}(y)} \neq \varnothing$, which implies that every continuous real-valued function of $T^{m}$ is constant on the set of isolated points of $T^{m-1}$ and, hence, it is constant on $T^{m-1}$ since this set is dense in $T^{m-1}$.

Finally, we consider the set $T=\bigcup_{m-1}^{\infty} T^{m}$ on which we define the following topology: If $t \in \mathbb{N}$, we first consider the basic open neighborhood $O_{U}^{1}(t)$ of $t$ in $T^{1}$ and then its corresponding basic open neighborhood in $T^{m}$,

$$
\begin{aligned}
O_{U}^{m}(t)=O_{U}^{m-1}(t) & \cup \bigcup_{n=1}^{\infty}\left(U\left(k^{m}(n)\right) \backslash\left\{k^{m}(n)\right\}\right) \\
& \cup \bigcup_{f_{m}\left(p^{m}(j)\right) \in O_{U}^{m-1}(t)}\left(U\left(p^{m}(j)\right) \backslash\left\{p^{m}(j)\right\}\right) .
\end{aligned}
$$

A basis of open neighborhoods of $t$ in $T$ is the collection of sets

$$
O_{U}(t)=\bigcup_{m=1}^{\infty} O_{U}^{m}(t), \quad U \in \mathscr{P} .
$$

If $t \in T \backslash \mathbb{N}$, then either $t \in X \backslash \mathbb{N}$ or $t$ is an isolated point of $T^{l}, l=1,2, \ldots$, where $l$ is the minimal integer for which $t \in T^{l}$.

In the first case, we first consider the basic open neighborhood $O_{U}^{1}(t)$ of $t$ in $T^{1}$ and then its corresponding basic open neighborhood in $T^{m}$,

$$
O_{U}^{m}(t)=O_{U}^{m-1}(t) \cup \underset{f_{m}\left(p^{m}(j)\right) \in O_{U}^{m-1}(t)}{\bigcup}\left(U\left(p^{m}(j)\right) \backslash\left\{p^{m}(j)\right\}\right) .
$$

A basis of open neighborhoods of $t$ in $T$ is the collection of sets

$$
O_{U}(t)=\bigcup_{m=1}^{\infty} O_{U}^{m}(t), \quad U \in \mathscr{P} .
$$

In the second case, we first consider the basic open neighborhood $O_{U}^{1}(t)$ of $t$ in $T^{l}$ and then its corresponding basic open neighborhood in $T^{l+m}$,

$$
O_{U}^{l+m}(t)=O_{U}^{l+m-1}(t) \cup \bigcup_{f_{l+m}\left(p^{l+m}(j)\right) \in O_{U}^{l+m-1}(t)}\left(U\left(p^{l+m}(j)\right) \backslash\left\{p^{l+m}(j)\right\}\right) .
$$

A basis of open neighborhoods of $t$ in $T$ is the collection of sets

$$
O_{U}(t)=\bigcup_{m=1}^{\infty} O_{U}^{m}(t), \quad U \in \mathscr{P} .
$$

From the definition of topology on $T$, it follows that, for every $t \in T$ and for every $U \in \mathscr{P}$, the set $O_{U}(t)$ is open-and-closed in $T \backslash \mathbb{N}$ and that $\overline{O_{U}(t)} \backslash O_{U}(t)=U$.

Proposition 1. The space $T$ is countable biconnected Hausdorff not widely connected and without a dispersion point.

Proof. That $T$ is countable Hausdorff is obvious. To prove that $T$ is connected, we consider two arbitrary points $x, y$ of $T$ and let $m$ be the minimal integer for which both $x, y \in T^{m}$. But then every continuous real-valued function of $T^{m+1}$ is constant on $T^{m}$ and, hence, for every continuous real-valued function $g$ of $T, g(x)=g(y)$, which implies that $T$ is connected.

Suppose now that $T$ is not biconnected and let $A, B$ be two connected, proper disjoint 
subsets containing more than one point and $A \cup B=T$. By the construction of the space $T$, it follows that $T \backslash \mathbb{N}$ is totally disconnected. Hence, there exists $b \in B \backslash \mathbb{N}$. Let $O_{U}(b)$ be the basic open neighborhood of $b$ defined by some $U \in \mathscr{P}$. Suppose that $\overline{O_{U}(b)} \cap B \cap \mathbb{N}=W \neq \varnothing$. If $W \notin \mathscr{P}$, then $\mathbb{N} \backslash W \in \mathscr{P}$ and, hence, for the set $O_{\mathbb{N} \backslash W}(b)$, it holds that $\overline{O_{\mathbb{N} \backslash W}(b)} \cap \mathbb{N}=\mathbb{N} \backslash W$. Therefore, $\overline{O_{U}(b) \cap O_{\mathbb{N} \backslash W}(b)} \cap B \cap \mathbb{N}=\varnothing$, which implies that the set $O_{U}(b) \cap O_{\mathbb{N} \backslash W}(b) \cap B$ is open-and-closed in $B$. Consequently, $B \subseteq O_{U}(b)$ for every $U \in \mathscr{P}$ and, hence, $B$ is a singleton, which is a contradiction. Hence, $W \in \mathscr{P}$. But then if we consider a point $a \in A \backslash \mathbb{N}$ and the basic open neighborhood $O_{U}(a)$ of $a$, it follows, in a similar manner, that the relation $\overline{O_{U}(a)} \cap A \cap \mathbb{N}=V \neq \varnothing$ implies that $V \in \mathscr{P}$, which is impossible because $B \cap A=\varnothing$. Therefore, either $\overline{O_{U}(a)} \cap A \cap \mathbb{N}=\varnothing$ or $\overline{O_{U}(b)} \cap B \cap \mathbb{N}=\varnothing$. Since $\overline{O_{U}(a)} \backslash O_{U}(a) \subseteq \mathbb{N}$ and $\overline{O_{U}(b)} \backslash O_{U}(b) \subseteq \mathbb{N}$, it follows that either $\overline{O_{U}(a)} \cap \mathbb{N}$ is open-and-closed in $A$ or $\overline{O_{U}(b)} \cap \mathbb{N}$ is open-and-closed in $B$. Hence, either the subset $A$ is a singleton or not connected, or the subset $B$ is a $\operatorname{singleton}$ or not connected.

That $T$ is not widely connected is obvious observing that, for every $U \in \mathscr{P}$ and every $t \in T$, the subset $\overline{O_{U}(t)}$ is connected. That $T$ has no dispersion point is obvious by its construction.

COROLLARY 1. The space $T$ is not strongly connected.

Proof. let $\tau$ denote the topology on $T$ and let $\tau_{\max }$ denote a maximal connected topology finer that $\tau$. By [13, Cor. $14 \mathrm{~A}]$, it follows that the space $\left(T, \tau_{\max }\right)$ has infinitely many cut points. Hence, if $t$ is such a point, then there exist two disjoint subsets $K$ and $L$ such that $K$ and $L$ are open-and-closed in $T \backslash\{t\}$, contain more than one point, and $K \cup L=T \backslash\{t\}$. Since the sets $K \cup\{t\}, L \cup\{t\}$, are connected in (T, $\left.\tau_{\max }\right)$, they are also connected in $(T, \tau)$. But by the proof of Proposition 1, it follows that, for every pair of connected subsets of $(T, \tau)$, which contain more than one point, their intersections include a member of $\mathscr{P}$. Therefore, the set $(K \cup\{t\}) \cap(L \cup\{t\})=\{t\}$ must be a member of $\mathscr{P}$, which is impossible.

COROLLARY 2. There exists $2^{c}$ mutually non-homeomorphic countable biconnected Hausdorff spaces not widely connected and without a dispersion point.

Proof. Because [19, Thm. 10], there exists $2^{c}$ different types of free ultrafilters on the discrete subspace $\mathbb{N}$ of the initial space $X$.

THE SPACE $S$. For the construction of the countable biconnected Urysohn almost regular space $S$, we first construct an appropriate countable Urysohn almost regular non-regular space and then, using the method of F. B. Jones [17], we construct a space $Y$ having the additional property of containing a point $\infty$ at which the space $Y$ is regular. The condensation process of this regular point is the same as in the construction of the space $T$.

We consider the initial space $X$ and, for every $n \in \mathbb{N}$, we consider a sequence $\left\langle b_{n i}\right\rangle_{i \in \mathbb{N}}$ converging to $n$ and consisting of isolated points not belonging to $X$. We set $B=\left\{b_{n i}\right.$ : $n, i=1,2, \ldots\}$ and we consider the space $C=X \cup B$. Let $C_{1}, C_{2}$ be disjoint copies of $C$ and let $p_{1}, p_{2}$ and $\mathbb{N}_{1}, \mathbb{N}_{2}$ be the copies of $p$ and $\mathbb{N}$ in $C_{1}, C_{2}$, respectively. We attach the space $C_{1}$ to $C_{2}$ identifying the point $p_{1}$ with $p_{2}$. We set $q=p_{1}=p_{2}$ and we consider the space $Z=\left(C_{1} \backslash\left\{p_{1}\right\}\right) \cup\{q\} \cup\left(C_{2} \backslash\left\{p_{2}\right\}\right)$ which is obviously Hausdorff but not regular 
since the point $q$ and the closed subset $\mathbb{N}_{1} \cup \mathbb{N}_{2}=K$ cannot be separated by disjoint open sets.

Let $Z_{n}, n=1,2, \ldots$ be disjoint copies of $Z$ and let $\bigcup_{n=1}^{\infty} Z_{n}$ be their disjoint union (topological sum). We add one more point $r$ and, on the set $L=\{r\} \cup \bigcup_{n=1}^{\infty} Z_{n}$, we define a basis of open neighborhoods of $r$ as follows: we consider the copies $B_{1}, B_{2}$ of $B$ in $C_{1}, C_{2}$, respectively. We set $B_{1} \cup B_{2}=R$ and let $R_{n}, n=1,2, \ldots$ be the copy of $R$ in $Z_{n}$. Let $\mathscr{R}$ be a free ultrafilter on the closed discrete subspace $Q=\left\{q_{n}: n=1,2, \ldots\right\}$, where $q_{n}$ is the copy of $q$ in $Z_{n}$. Then, for every $U \in \mathscr{R}$, a basis of open neighborhoods of $r$ is the collection of sets $U(r)=\{r\} \cup\left\{\cup R_{i}: q_{i} \in U\right\}$.

It can be easily verified that the space $L$ is Urysohn but not normal since the closed subsets $Q$ and $\bigcup_{n=1}^{\infty} K_{n}$, ( $K_{n}$ is the copy of $K$ in $Z_{n}$ ) cannot be separated by disjoint open sets. Also, the subsets $\bigcup_{n=1}^{\infty} K_{n}$, and the point $r$ cannot be separated by disjoint open sets, while $Q$ and $r$ can be separated by disjoint open sets but not by disjoint open-and-closed sets. However, $L$ is not regular at $r$. Since the closed subsets $Q$ and $\{r\}$ of $L$ cannot be separated by a continuous real-valued function, it follows that if we consider $L_{n}, n=1,2, \ldots$ disjoint copies of $L$, we can apply the construction in [17] and obtain a space $Y$ with the following properties

(1) It is countable Urysohn containing a dense subset of isolated points.

(2) It contains a point $\infty$ at which $Y$ is regular.

(3) The point $\infty$ and each copy $Q_{n}, n=1,2, \ldots$ of the subset $Q$, in $L_{n}$ cannot be separated by disjoint open-and-closed subsets, that is they cannot be separated by a continuous real-valued function of $Y$.

Proposition 2. There exists $2^{c}$ mutually non-homeomorphic countable biconnected Urysohn almost regular spaces, not widely connected, not having a dispersion point, and not being strongly connected.

Proof. We imitate the condensation process that we used in the construction of the space $T$ using the space $Y$ in place of the space $X$ and the point $\infty$ and the set $Q_{1}$ in place of $p$ and $\mathbb{N}$, respectively. Let $S^{m}, m=1,2, \ldots$ and $S=\bigcup_{m=1}^{\infty} S^{m}$ be the corresponding spaces to $T^{m}$ and $T$, respectively. It can be easily proved that $S$ is Urysohn. Since the different copies of the regular point $\infty$ are attached in each step to the isolated points of $S^{m}, m=1,2, \ldots$, it follows that these points remain regular in the final space $S$. Obviously, the set of all these points is dense in $S$ and, hence, $S$ is almost regular.

All the other properties of $S$ are proved as in Proposition 1 and Corollaries 1 and 2.

REMARK. In [37], E. K. van Dowen constructed a regular space with a dispersion point on which every continuous real-valued function is constant. We can modify his method and construct a countable biconnected Hausdorff space not widely connected, not having a dispersion point, and not being strongly connected. For this, we consider again the initial space $X$ and let $X_{i}, i=1,2, \ldots$ be disjoint copies of $X$. We denote by $x_{i}$ the copy of $x \in X$ in $X_{i}$, and by $\mathbb{N}_{i}$ the copy of $\mathbb{N}$. We attach the spaces $X_{i}, i=2,3, \ldots$ to $X_{1}$ identifying each copy $\mathbb{N}_{i}$ with $\mathbb{N}_{1}$, that is by putting each $n_{i}$ to $n_{1}$. We denote this point by $n$. In the space $Z=\mathbb{N} \cup \bigcup_{i=1}^{\infty}\left(X_{i} \backslash N_{i}\right)$, the subset $P=\left\{p_{i}: i=1,2, \ldots\right\}$ and the subset $D$ consisting of all isolated points of the copies $X_{i}$ are countable and, 
therefore, there exists a one-to-one function $g$ of $P$ onto $D$. On the quotient space $T_{X}=\mathbb{N} \cup\left\{\left(p_{i}, g\left(p_{i}\right)\right): i=1,2, \ldots\right\}$, we define a second topology $\tau$ in a similar manner as in the construction of the space $T$. Obviously, the topology $\tau$ is weaker than the quotient topology of $T_{X}$. It can be proved, as in Proposition 1 and Corollaries 1 and 2, that $\left(T_{X}, \tau\right)$ is the required space.

In a similar manner, we can construct a Urysohn almost regular space having all the above properties. For this, it suffices to consider space $Y$ as the initial space.

ACKNOWLEDGEMENT. The author is grateful to the referee for his suggestions and comments.

\section{REFERENCES}

[1] I. Baggs, A connected Hausdorff space which is not contained in a maximal connected space, Pacific J. Math. 51 (1974), no. 1, 11-18. MR 51 1729. Zbl 295.54046.

[2] J. Cobb and W. Voxman, Dispersion points and fixed points, Amer. Math. Monthly 87 (1980), no. 4, 278-281. MR 81e:54036. Zbl 434.54038.

[3] S. F. Cvid, A countable strongly unicoherent space, Mat. Zametki 24 (1978), no. 2, 289-294, 303 (Russian), (Engl. translation: Math. Notes (1-2)(1978), 655-657). MR 80b:54045. Zbl 409.54046.

[4] R. Duda, On biconnected sets with dispersion points, Rozprawy Mat. 37 (1964), 60. MR 28\#5412. Zbl 141.20402.

[5] A. G. El'kin, Maximal connected Hausdorff spaces, Math. Notes 26 (1979), no. 5-6, 974978, (Translated from Matem. Zametki 26 (1979), no. 6, 939-948. MR 81c:54028). Zbl 435.54018.

[6] P. Erdös, Some remarks on connected sets, Bull. Amer. Math. Soc. 50 (1944), 442-446. MR 6,43a.

[7] G. Gruenhage, Spaces in which the nondegenerate connected sets are the cofinite sets, Proc. Amer. Math. Soc. 122 (1994), no. 3, 911-924. MR 95j:54024. Zbl 813.54015.

[8] C. Guido, Connected spaces which are not strongly connected, Rend. Circ. Mat. Palermo (2) (1984), no. 6, 149-153, Proceedings of the 12th winter school on abstract analysis (Srni 1984). MR 54 1165. Zbl 565.54014.

[9] W. Gustin, Countable connected spaces, Bull. Amer. Math. Soc. 52 (1946), 101-106. MR 7,334g. Zbl 060.39606.

[10] A. Gutek, On biconnected spaces without dispersion points, Comment. Math. Prace Mat. 21 (1980), no. 1, 63-70. MR 82f:54083.

[11] _ A connected space with a dispersion point without fixed-point property, Topology Appl. 30 (1988), no. 1, 101-105. MR 89k:54087. Zbl 661.54038.

[12] J. A. Guthrie, D. F. Reynolds, and H. E. Stone, Connected expansions of topologies, Bull. Austral. Math. Soc. 9 (1973), 259-265. MR 48 7201. Zbl 261.54002.

[13] J. A. Guthrie and H. E. Stone, Spaces whose connected expansions preserve connected subsets, Fund. Math. 80 (1973), 91-100. MR 48 9653. Zbl 271.54014.

[14] J. A. Guthrie, H. E. Stone, and M. L Wage, Maximal connected expansions of the reals, Proc. Amer. Math. Soc. 69 (1978), 159-165. MR 57 7501. Zbl 396.54001.

[15] P. C. Hammer and W. E. Singletary, Connectedness-equivalent spaces on the line, Rend. Circ. Mat. Palermo (2) 17 (1968), 343-355. MR 43\#8054. Zbl 197.19003.

[16] S. Iliadis and V. Tzannes, Spaces on which every continuous map into a given space is constant, Canad. J. Math. 38 (1986), no. 6, 1281-1298. MR 87m:54109. Zbl 599.54043.

[17] F. B. Jones, Hereditarily separable, non-completely regular spaces, Topology Conference (Virginia Polytech. Inst. and State Univ., Blacksburg, Va., 1973) (Berlin), Lecture Notes in Math., vol. 375, Springer-Verlag, 1974, pp. 149-152. MR 541165. Zbl 286.54008. 
[18] V. Kannan, A countable connected Urysohn space containing a dispersion point, Proc. Amer. Math. Soc. 35 (1972), no. 1, 289-290. MR 45 5967. Zbl 262.54009.

[19] V. Kannan and M. Rajagopalan, Regularity and dispersion in countable spaces, Duke Math. J. 39 (1972), 729-734. MR 46 9928. Zbl 263.54009.

[20] _ Constructions and applications of rigid spaces. I, Adv. in Math. 29 (1978), no. 1, 89-130. MR 82e:54045a. Zbl 403.54030.

[21] H. Katsuura, Dispersion points and continuous functions, Topology Appl. 28 (1988), no. 3, 233-240. MR 89c:54089. Zbl 642.54032.

[22] J. R. Kline, A theorem concerning connected points sets, Fund. Math. 2 (1922), 238-239.

[23] B. Knaster and K. Kuratowski, Sur les ensembles connexes, Fund. Math. 2 (1921), 206-255.

[24] K. Kuratowski, Topology, vol. I, Academic Press, New York, London, 1966. MR 36\#840. Zbl 158.40802 .

[25] J. Martin, A countable Hausdorff space with a dispersion point, Duke Math. J. 33 (1966), 165-167. MR 33\#699. Zbl 137.16003.

[26] E. W. Miller, Concerning biconnected sets, Fund. Math. 29 (1937), 123-133. Zbl 017.13402.

[27] G. G. Miller, A countable Urysohn space with an explosion point, Notices Amer. Math. Soc. 13 (1966), 589.

[28] _ Countable connected spaces, Proc. Amer. Math. Soc. 26 (1970), 355-360. MR 41\#7610. Zbl 198.55503.

[29] P. Roy, A countable connected Urysohn space with a dispersion point, Duke Math. J. 33 (1966), 331-333. MR 33\#4887. Zbl 147.22804.

[30] M. E. Rudin, A connected subset in the plane, Fund. Math. 46 (1958), 15-24. MR 20\#4817. Zbl 227.54029.

[31] _ A biconnected set in the plane, Topology Appl. 66 (1995), 41-48. MR 96m:54031. Zbl 831.54003.

[32] P. Simon, An example of maximal connected Hausdorff space, Fund. Math. 100 (1978), no. 2, 157-163. MR 58 7557. Zbl 435.54017.

[33] P. M. Swingle, Generalizations of biconnected sets, Amer. Journal of Math. 53 (1931), 385400.

[34] _ Two types of connected sets, Bull. Amer. Math. Soc. 37 (1931), 254-258. Zbl 001.35404 .

[35] J. P. Thomas, Maximal connected topologies, J. Austral. Math. Soc. 8 (1968), 700-705. MR 38\#5177. Zbl 165.25302.

[36] V. Tzannes, A countable widely connected Hausdorff space, Topology Appl. 69 (1996), 63-70. MR 97a:54021. Zbl 858.54034.

[37] E. K. van Dowen, A regular space on which every continuous real-valued function is constant, Nieuw Arch. Wisk. 20 (1972), no. 3, 143-145. MR 47 1019. Zbl 237.54030.

[38] E. J. Vought, A countable connected Urysohn space with a dispersion point that is regular almost everywhere, Colloq. Math. 28 (1973), 205-209. MR 48 4998. Zbl 264.54013.

[39] R. Wilder, A point set which has no true quasicomponents and which becomes connected on the addition of a single point, Bull. Amer. Math. Soc. 33 (1927), 423-427.

TZANnes: Department of MAthematics, University of Patras, Patras 26110, Greece 


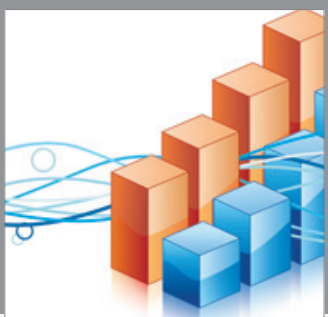

Advances in

Operations Research

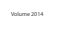

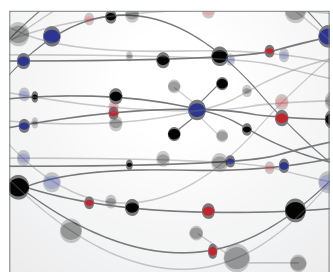

\section{The Scientific} World Journal
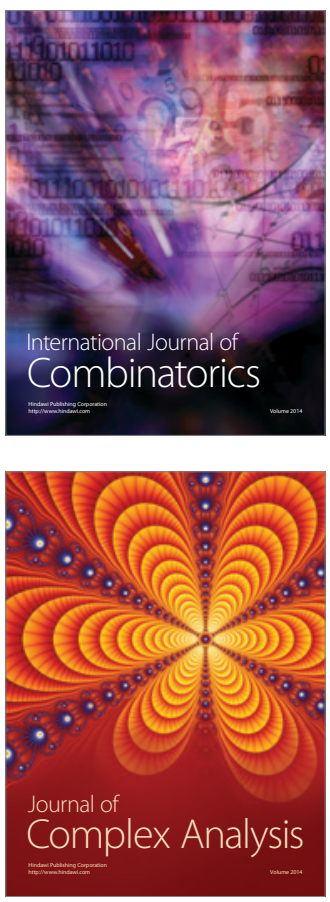

International Journal of

Mathematics and

Mathematical

Sciences
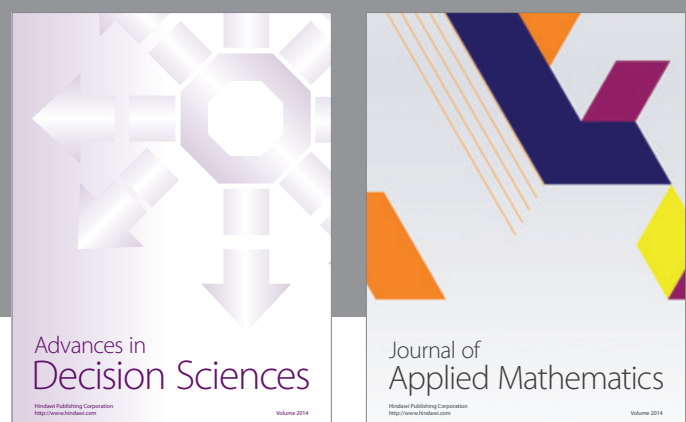

Journal of

Applied Mathematics
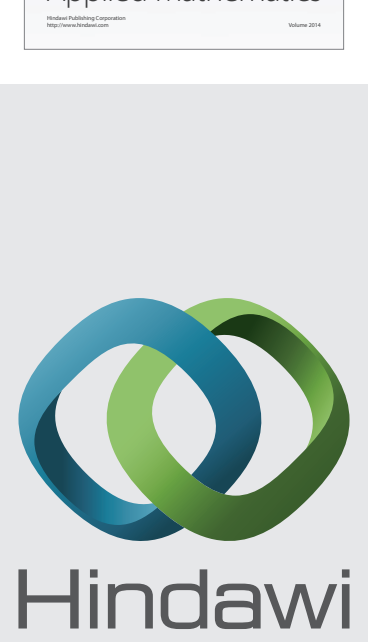

Submit your manuscripts at http://www.hindawi.com
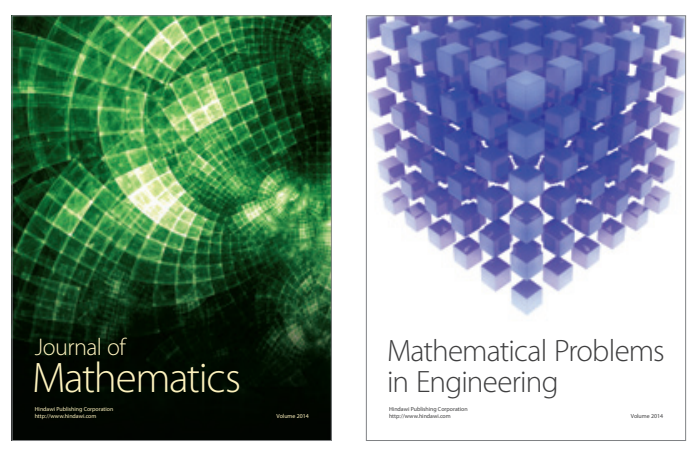

Mathematical Problems in Engineering
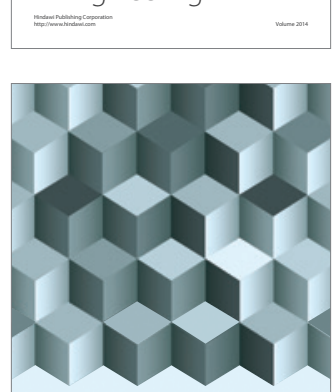

Journal of

Function Spaces
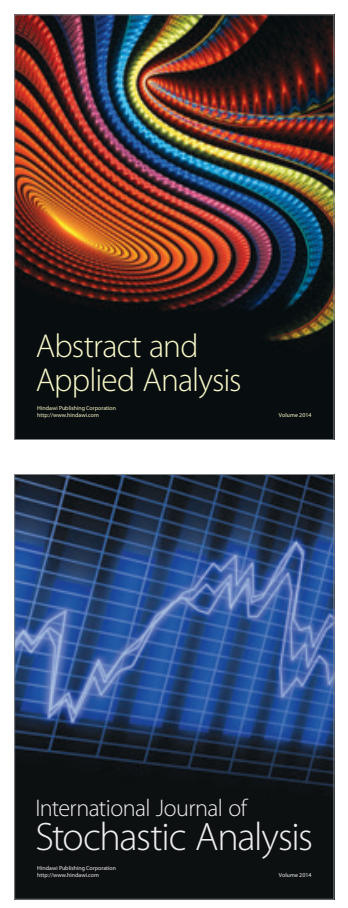

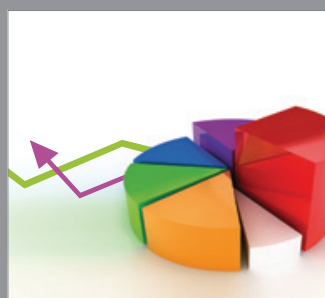

ournal of

Probability and Statistics

Promensencen
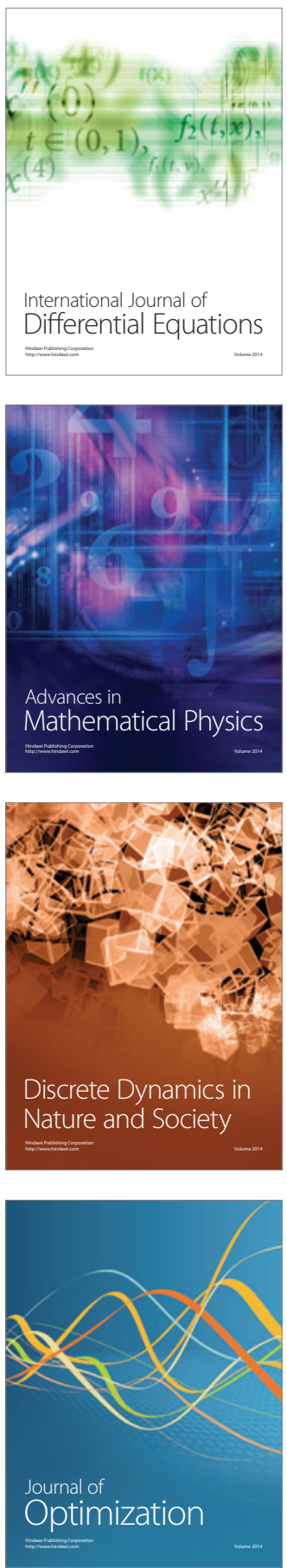